\title{
VIRTOOL - Virtual Reality for Machine - Tool Training
}

\author{
Aritz Ustarroz ${ }^{1, a}$, Alberto Lozano $^{1}$, Luis Matey ${ }^{1,2}$, Jens Siemon $^{3}$, Diana Klockmann ${ }^{3}$ \\ and Miren Iosune Berasategi ${ }^{4}$ \\ 1 CEIT (Centro de Estudios e Investigaciones Técnicas de Gipuzkoa), Lardizabal 15, 20018 San Sebastián, Spain \\ 2 University of Navarra, Lardizabal 13, 20018 San Sebastián, Spain \\ 3 TUD (Dresden University of Technology), Münchner Platz 1, 0062 Dresden, Germany \\ 4 IMH (Instituto de Máquina Herramienta), San Roke 1, 20870 Elgoibar, Spain
}

Received 30 June 2003, Accepted 20 October 2003

\begin{abstract}
VIRTOOL is an application that is part of the European Union supported project "Virtual Manipulation to Simulate Machine-Tool Processes" (VIRTOOL, CRAFT-1999-70292). The application is being developed by a consortium of two industrial enterprises (Alecop, IMH) and three partners in research and higher education (CEIT, TUD, ACM) from four European countries. The objective of this project is to design and develop a computer-supported learning environment for machine-tool processes by using interactive 3D graphics and virtual reality techniques. An integral part of this software will be an easy to use construction tool for the development of new 3D simulation models of machine-tools with the supporting learning material, which is based on a general instructional design model.
\end{abstract}

Key words: Virtual reality / machine-tool / real time visualization / case studies / instructional design

\section{Introduction}

In many cases, the limitation of resources and the prevention of risks in industrial formation environments imply that the teaching does not have the desired quality. If classes are imparted to too many students, often it is not possible that all could practice with the real machinery by reason of limitation of time, space, or because the acquisition of the machine is not profitable for the educational institution. Another reason that implies the need of new teaching-tools lies in the impossibility of using the machinery in limit situations or allowing the self-directed learning, because it is a risk for the security of the student and the integrity of the machinery. For these motives, many times the explanations are limited to theoretical classes, and it implies that the correct evaluation of the students' skills is not completely guaranteed.

Thanks to the advance of the computers, new possibilities appear in order to complement the practice with real machinery. The VR systems seem to be very adequate for the educational environments. These systems get ready the student before working with the real machinery. They put them in situations that are going to meet later without fear to probable errors and they have the opportunity of working the necessary time in order to acquire assurance. When these students use the real machinery they

\footnotetext{
${ }^{a}$ Corresponding author: austarroz@caf .es
}

will identify the actions that should execute quickly in order to resolve the specific task. Also, VR systems offer the necessary flexibility in order to practice with machinery that the educational institution does not provide, and therefore they offer the student more knowledge than the theoreticals.

VR systems themselves already are of great utility, but they will be even more if they are complemented with learning materials. An Intelligent Tutoring System (ITS) could guide the process of learning, identify the errors that the student commits and offer you the learning resources in order to solve them. The ITS is used with proved efficacy in fields like flight-training $[1,2]$ or the training for maintenance tasks [3] besides others. Also, applications like FROGALAN [4] have demonstrated to be useful in machine-tooling, since it permits to diagnose the knowledge of the student from a practical point of view.

\section{Project objective}

The objective of the VIRTOOL project is to design and develop a computer-supported learning environment for machine-tool (conventional and CNC) processes by using interactive 3D graphics and virtual reality techniques. Actually there are many virtual reality applications to manipulate systems but they are not completely 
suitable to machine-tool operations. This tool specially focuses on the training aspects relating to machine-tool. However, the software integrates an easy to use preprocessing part that helps defining and generating new models of machine-tool and digital libraries of accessories. New learning material can be prepared in order to build a complete database of learning stages [5].

There are three main issues that can make the use of VIRTOOL relevant. First of all, the cost of the machines, which are expensive, secondly the availability of these machines (normally difficult for educational centres due to limitations of space and cost, etc.) and last but not least safety conditions. These reasons and the lower cost of new virtual reality equipment are ideal conditions, which favour the educations of students with VIRTOOL.

VIRTOOL-simulation machines offer the learner a broad range of different interactions by using two- and tri-dimensional human-computer-interfaces [6,7]. The interaction alternatives could be divided in the set-up of the machine-tool and the machining itself. In the machinetool set-up, the learners always have more than one alternative to perform the operations of greasing and lubrication, loading workpiece plan file, mounting the workpiece trunnion tooling or mounting the cutting tool.

Also in machining, VIRTOOL-machines offer the learner different possibilities to define the machining process, mount the workpiece, select parameters, orientate the cutting tool, select the security elements, set-up the tool, operate the machine, measure the workpiece and finish the process.

Errors will occur as naturally as they occur in real live machinery and will be simulated as dramatically as it is technically possible to show the learner the possible results of misconceptions or wrong operations. But there are two general differences between real live machining and maximum authentic VR-simulations. First, nothing happens physically and second, it is probable that the cognitive processes of the learners are enhanced and positively supported.

Currently, the development of the software has not finished yet (it is supposed to finish in 2003). VIRTOOL Master is nearly finished and VIRTOOL Generic is close to a very advanced prototype version. On the other hand, VIRTOOL Machine gathers many of the functions described in this paper but it is a basic prototype version.

\section{Software description}

\subsection{Introduction and schema of the system architecture}

VIRTOOL is a software tool formed by four applications: Master, Generic, Case Generator and Machine. The first three applications constitute the preprocessing step. Firstly, VIRTOOL Master generates machine-tool models, both conventional and CNC, that is, it builds the kinematic definition for each machine and assigns this definition to the different assemblies or parts of the whole machine. The geometric parts are imported in VRML 2.0 standard format from CAD applications. On the other hand, VIRTOOL Generic allows machine-tools manufacturers and professors to generate specific elements or accessories libraries for different machines. Cutting tools, holders and fixtures, mounting tools or measurement tools are some examples of these accessories. The last application of the pre-processing step is VIRTOOL Case Generator, where teachers produce cases or exercises according to the learners' knowledge and generate the knowledge database that later will provide feedback in VIRTOOL Machine.

VIRTOOL Machine, which is based on virtual reality techniques, is the final application to be used in the training process. This application offers the learners a wide range of different interactions by using two and threedimensional computer interfaces. It imports the machine and elements definition from Master and Generic, and the case information from VIRTOOL Case Generator.

The software of VIRTOOL project is developed using Microsoft Visual $\mathrm{C}++$ and Microsoft Windows 2000 OS. VIRTOOL Master, Generic and Machine also use OpenGL graphical library for the visualization of $3 \mathrm{D}$ environments.

Figure 1 shows the schema of the system architecture.

\subsection{Description of the applications}

\subsubsection{VIRTOOL Master}

VIRTOOL Master application's main function is to define machine-tools specific models. CAD files are preferably imported in VRML 2.0 standard format, although other formats like VRML 1.0 or STL are also supported. Every CAD file represents any part of the machine and the user (engineers and manufacturers with technical overview) builds the geometry of the machine grouping each machine part in different assemblies. These geometrical relationships are stored in a XML file.

The next step is to generate a XML file with the kinematics of the model, taking into account the different kinematic elements (bodies) and their relationship and dependence (joints). It is possible to produce any machine-tool kinematics providing that it is an openchain kinematics. Subsequently, the user can build the machine step by step, assigning kinematic properties to machine's assemblies and watching the result on a $3 \mathrm{D}$ graphic window.

As a result, VIRTOOL Master produces a final XML file with the complete machine definition. The previously described procedure can be seen in Figure 2.

\subsubsection{VIRTOOL Generic}

VIRTOOL Generic is thought to be a very useful application for both manufacturers and teachers from manufacturing schools. It allows generating a complete digital library of machine-tool elements or accessories, such as cutting tools, tool holders and fixtures, mounting tools, 


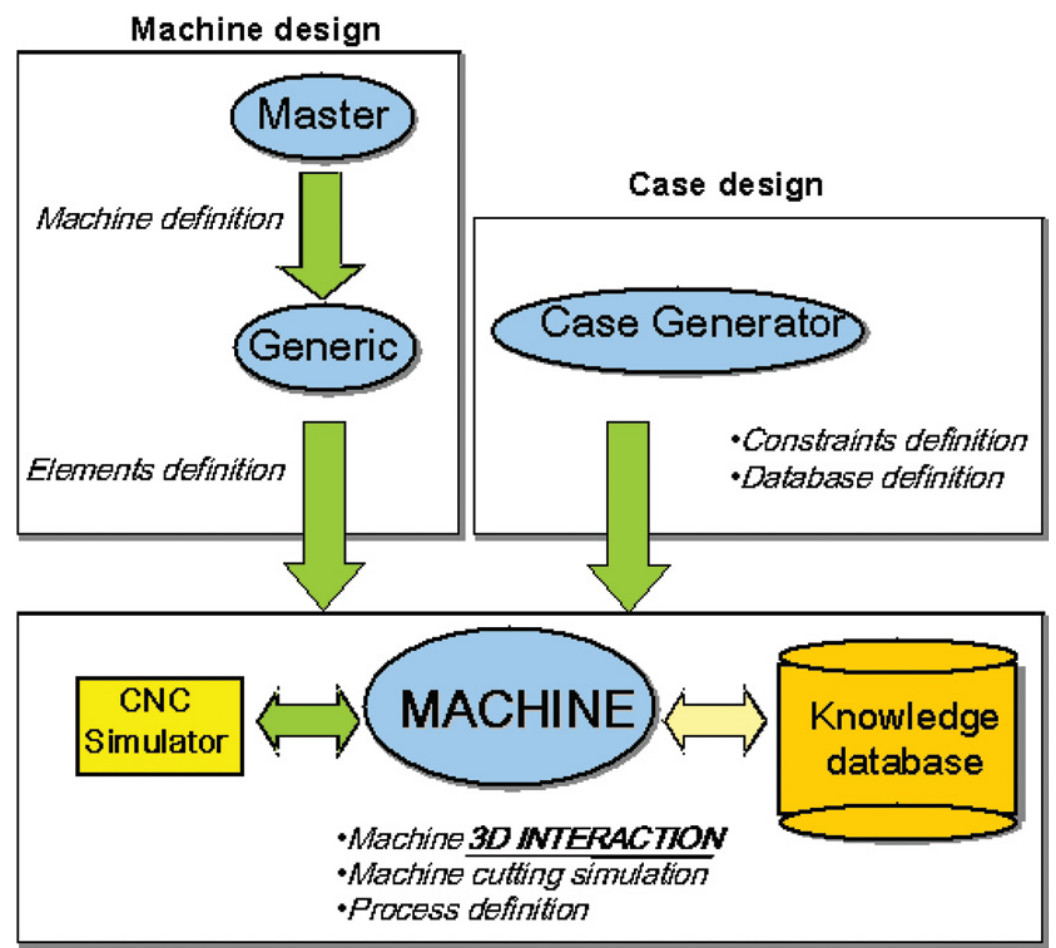

\section{Learning \& training process}

Fig. 1. System architecture schema.

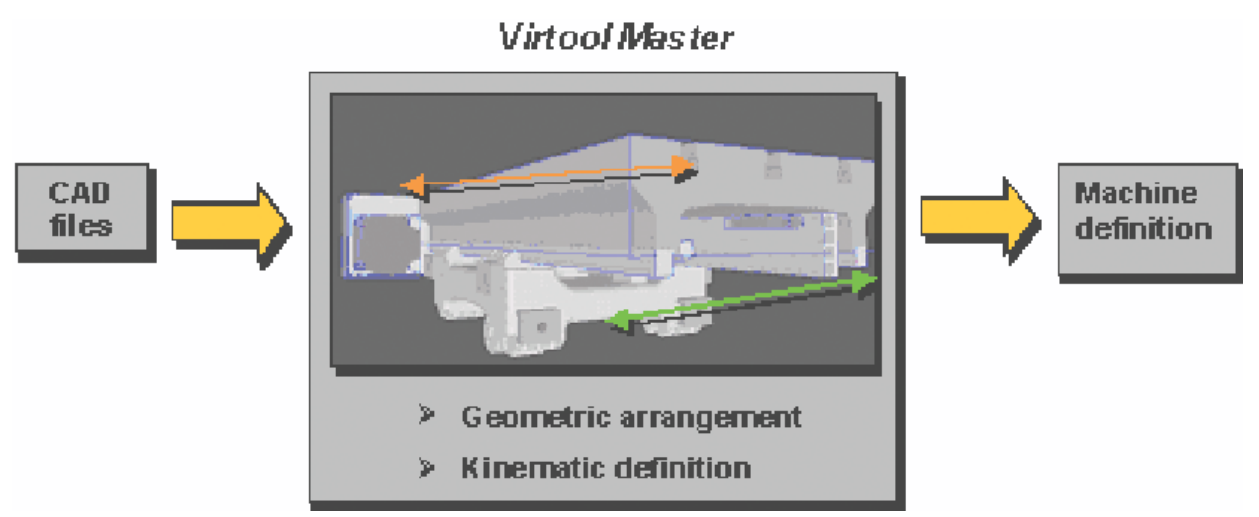

Fig. 2. VIRTOOL Master description.

measurement hand tools and lubricating and coolant oils. The main benefit provided by VIRTOOL Generic is the possibility to introduce several elements from different manufacturers and to finally obtain a digital library where learners can choose between almost all the cutting tools that are sold in the market or between different clamping systems. The VIRTOOL Generic schema is depicted in Figure 3.

The user can define the necessary parameters of each element while he can view the correspondent $3 \mathrm{D}$ model and a 2D schematic drawing (useful when defining cutting tools for example). The machine-tool 3D model can be visualized in another window, although all its properties were previously defined in VIRTOOL Master. VIRTOOL Generic produces a final XML file, where elements properties are stored.

\subsubsection{VIRTOOL Case Generator}

With this software-tool, content experts such as teachers, textbook-authors or curriculum-designers can generate special cases or series of cases according to the content, the knowledge of the students, their abilities and the machine properties, which are defined in VIRTOOL Master and Generic.

But also machine-tool manufacturers are able to create special cases, even for further vocational training of their employees, to underline the special abilities and strengths of a particular machine, or to make rather complex product more transparent.

In Figure 4, the main functions of VIRTOOL Case Generator are summarised. The user, commonly a teacher, states which are the main objectives of the 


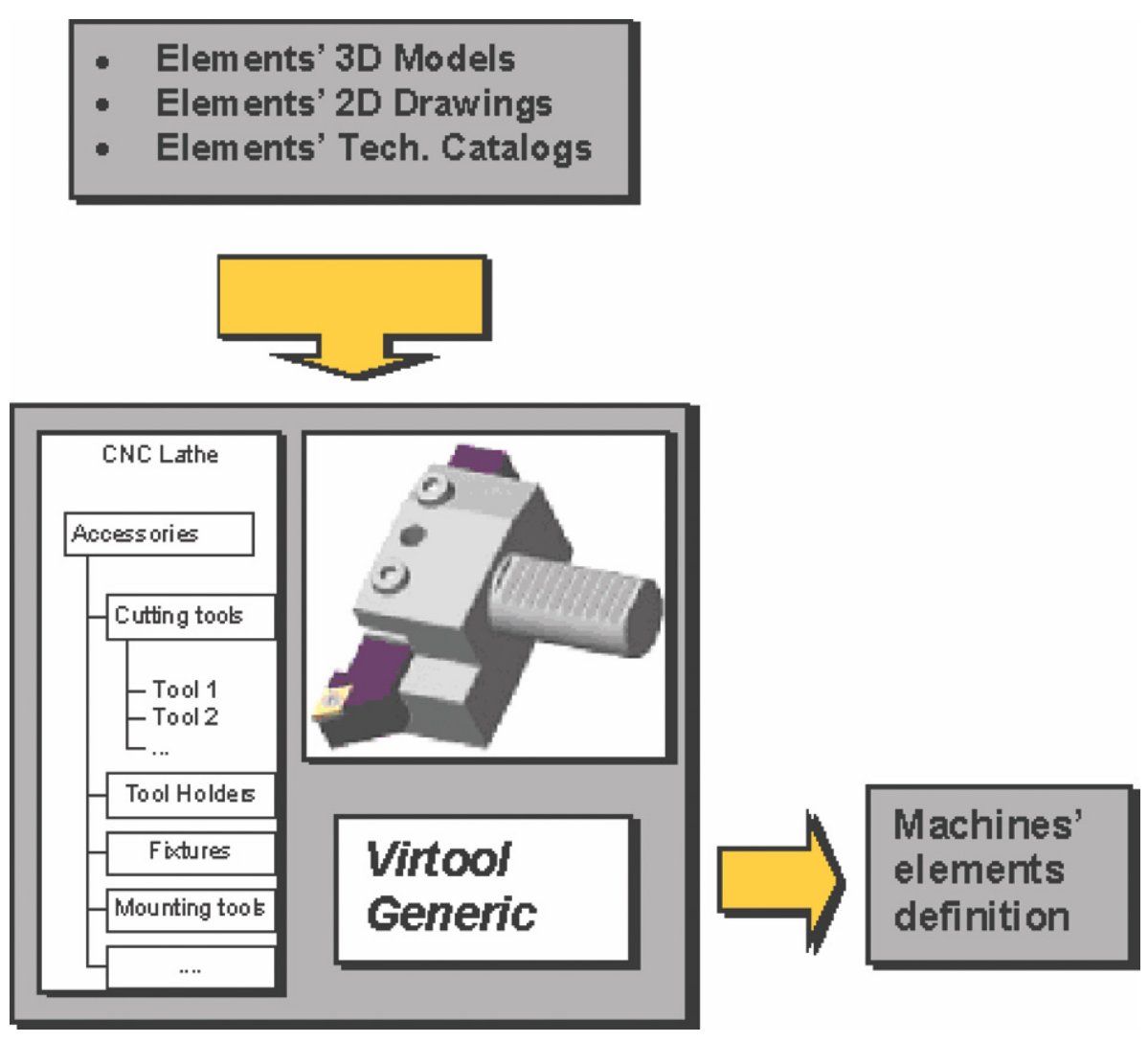

Fig. 3. Concept of VIRTOOL Generic.

exercise and introduces a $2 \mathrm{D}$ drawing of the required workpiece. Some additional material is also added to the case in order to help learners when they must work with the machine within the virtual environment; tutorials and demos (videos) are part of this help material.

Last but not least, it is important to underline that VIRTOOL Machine is not a machine-tool dynamic simulator and thus, some extra information, based on teacher's experience, should be included in the case's information so as to produce errors and the subsequent feedback. Hence, VIRTOOL Case Generator provides the possibility to define allowed and forbidden operations and their effects. This list can be stored for following cases.

\subsubsection{VIRTOOL Machine}

VIRTOOL Machine is the final application to be used in the training process. Teachers will use the tool to prepare different training exercises for a specific machine-tool and adapt it to their students' needs. The students themselves will receive specific training for a real machine by using virtual machine simulations. Figure 5 depicts VIRTOOL machine's concept, which is described in the following lines.

VIRTOOL Machine is a desktop VR system, where the user interacts with the virtual environment by using a $2 \mathrm{D}$ mouse or a 3D mouse and receives stereo visualization and sound feedback. VIRTOOL Machine is thought to be a low cost application, since it might be mostly used in classrooms. In addition to the visual and manipulation modules, the other principal module is the collision detection module.

This application imports all the data that have been previously generated in VIRTOOL Master, Generic and Case Generator. Machine-tool 3D models information is received from Master, while the properties of the accessories and cases' information arise from Generic and Case Generator respectively. This cases' information implies the establishment of a knowledge database.

Although material removal simulation was not one of the project's objectives, the consortium noticed the possibility to integrate the CNC simulator WinUnisoft [8] whose owner is Alecop. This integration has not been performed yet, but due to the modularity of the CNC simulator, there is a serious possibility to include the simulation even in the main environment of VIRTOOL Machine.

Feedback gain importance since VIRTOOL Machine is focused on learning and training processes in classrooms and in manufacturing factories. In addition to visual and sound feedback, VIRTOOL Machine also produces some data feedback. Different errors might be happened during the machine's manipulation and the feedback to these errors may be visualized in the $3 \mathrm{D}$ window (e.g. fixtures or tool release, tool fracture) or may be loomed like error messages suggesting possible causes and solutions. Automatic evaluation is also a very useful feedback tool to give learners the result of their performance. 


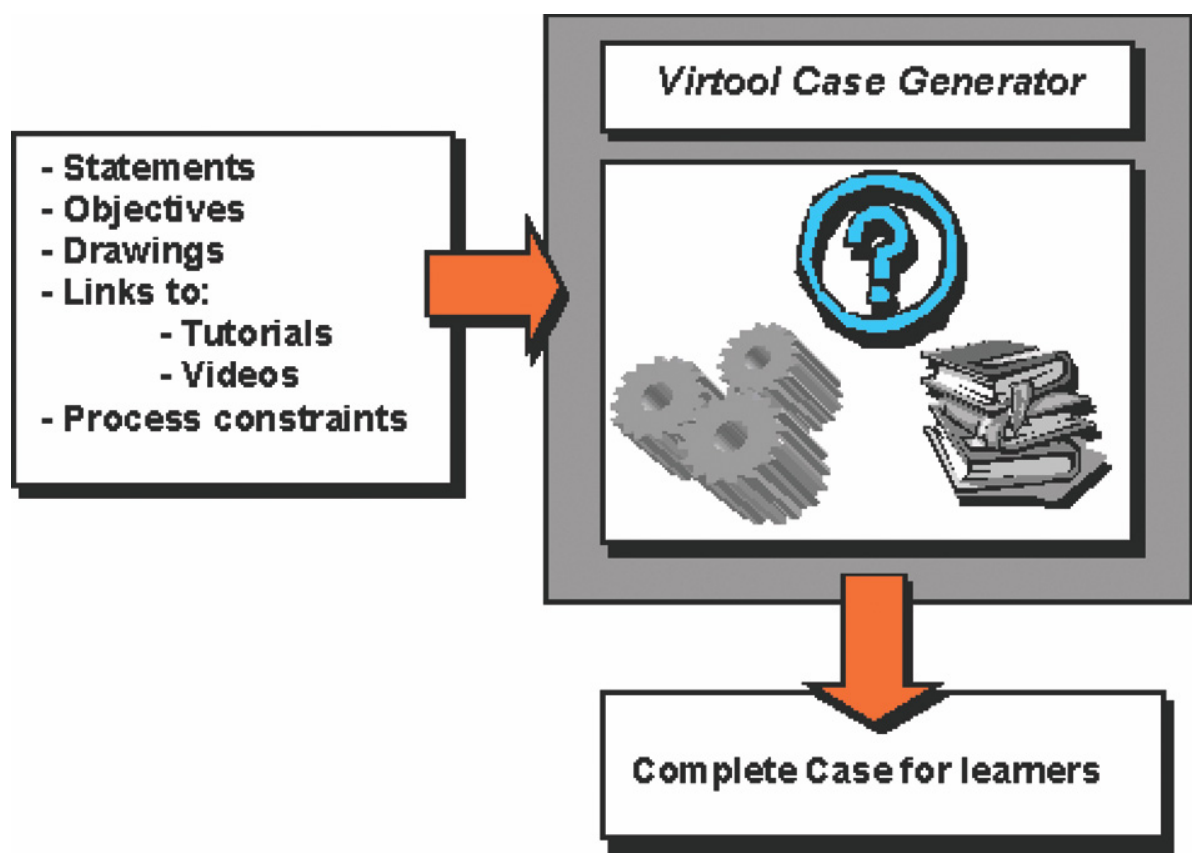

Fig. 4. VIRTOOL Case Generator.

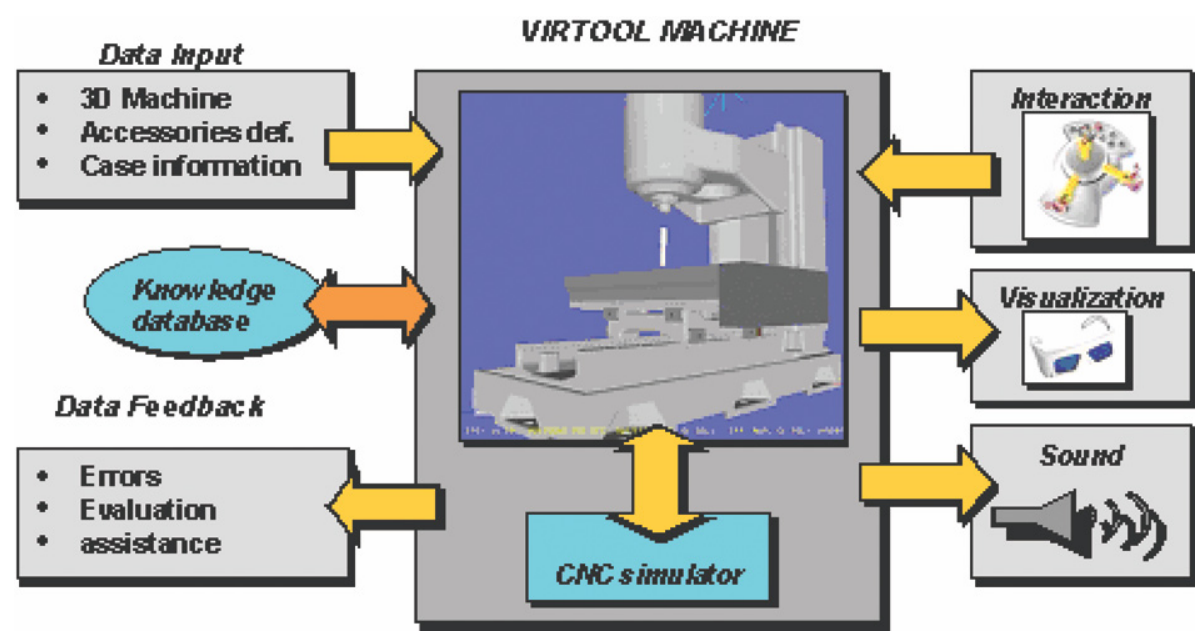

Fig. 5. Architecture of VIRTOOL Machine.

\section{Instructional design}

The creation of a workpiece is generally characterised by a fixed procedure that is defined by a different number of decisions, different levels of complexity or sub-decisions and alternatives. The basic procedure remains the same and should therefore build an anchor for the learners to gain insight into the different areas of the professional skills.

As students need to understand this basic scheme, an expert can be provided to show the students his procedures when creating a workpiece and to make his internal thinking processes visible. Psychologists call this the externalisation of tacit knowledge [9, 10].

In the classroom, normally the teacher takes over this part with the help of computer-based learning or teaching videos. The latter enable the students to rewind and to have a closer look at particular sequences and therefore to revise more deeply.

The video where knowledge is embedded can be stopped and replayed individually, a procedure that has proved to be successful in many classroom environments. Broad research results for technical professions have yet to be provided $[11,12]$. The students are provided with a broad selection of tasks to be solved. The single tasks equal the production of workpieces, as demanded in the professional requirements, which are supported by the learning environments. Every single task is designed like a case study by applying holistic, activity-oriented approaches.

Case studies can be worked on in any order and choice. There are studies with appropriate levels for each lesson content. Furthermore, it is planned to introduce 
transfer cases where students can use the knowledge in different areas and the use of knowledge outside the working world or the specific learning situation. The construction tool Case Generator offers the opportunity to enlarge the number of cases and to create more learning material. This ensures that teachers in the classroom can choose the case that suits best for the lesson and work on it with the students.

Research in the area of self-directed learning has shown that beginners learn more effectively when some guiding is provided for them, whereas learning through trial and errors is not suitable in early learning stages $[13,14]$. A self-study mode is therefore installed to suggest the learning sequences which are useful for further self-directed learning.

With the aim of improving the learning system, Artificial Intelligence techniques are being evaluated. The learning process of every student is not the same. There are concepts that some students learn quicker than others. Therefore, it would be adequate to adapt the system to them. This is feasible by embedding an Intelligent Tutoring System (ITS) in VIRTOOL that customizes the teaching with each student model. The students' skills are evaluated by means of specific tasks to resolve. In test situations, similar cases to the ones of the learning sequences are used. Learners should identify the type of given task and apply the correct knowledge structures in order to resolve it successfully. The ITS verifies the students' understanding and shows them a suitable learning material according to the evaluation result. The ITS is a good way to help human instructors in learning processes.

A special interest is also given to the evaluation processes. During the learning phases, it is possible for the student to self-evaluate every single step or decision by simulating its typical desired or non-desired results. The effects will be shown to the student by visual and audible simulation of the machine, the tool movements and the changes of the workpiece-shape. But it will also be possible to simulate the destruction of the workpiece, the tool, or the entire machine itself. These evaluation processes are analysed by the ITS, which updates the student model for resolving the next learning step.

Therefore, Case Generator is the responsible of creating learning material and ITSs are computer-based instructional systems with models of instructional content that specify what to teach, and teaching strategies that specify how to teach $[15,16]$.

\section{Conclusions}

\section{VIRTOOL is a software which can}

- Make training processes in initial and further vocational education more efficient by using a state of the art instructional design based on the latest psychological and pedagogical findings and on the latest technology, e.g. VR and 3D-interaction devices.

- Reduce the risk for accidents during the training processes when using powerful machine-tools.
- Reduce the costs for educational processes.

- Increase the availability of machines that are normally not used by educational centres because of its specialisation.

- Help machine-tool manufacturers to explain their complex and complicated products.

\section{References}

[1] R.A. Richards, Principle Hierarchy Based Intelligent Tutoring System for Common Cockpit Helicopter Training, ITS 2002 Proceedings, 2002, pp. 473-483

[2] M. Aka, C. Frasson, ASIMIL: Overview of a Distance Flight-Training System ITS 2002 Proceedings, 2002, pp. $484-495$

[3] R. Stiles, L. Johnson, A. Munro, VET: Virtual Environments for Training, http://btl.usc.edu/VET/, 1998

[4] B. Ferrero, I. Fernández-Castro, M. Urretavizcaya, Diagnostic et évaluation dans les systèmes de "training" industriel, Diagnosis and assessment in industrial training systems, Simulation et formation professionnelle dans l'industrie, 6(1) (1999), 189-217

[5] M. Shelbourn, G. Aouad, M. Hoxley, Multimedia in construction education: new dimensions, in Automation in Construction, 10, 2001, 265-274

[6] M. Weyrinch, P. Drews, An interactive environment for virtual manufacturing: the virtual workbench, Computers in Industry, 38, Elsevier, 1999

[7] W.B. Lee, C.F. Cheung, J.G. Li, Applications of virtual manufacturing in materials processing, Journal of Materials Processing Technology, 113(1-3) (2001), 416-423

[8] WinUnisoft CNC simulator. www.alecop.es

[9] R.M. Gagné, E.C. Smith, A Study of the Effects of Verbalization on Problem Solving, Journal of Experimental Psychology, 63 (1962), 12-18

[10] A. Collins, J. Brown, S.E. Newman, Cognitive Apprenticeship: Teaching the Crafts of Reading, Writing, and Mathematics, in L.E. Resnick (ed.), Knowing, Learning and Instruction - Essays in Honor of Robert Glaser, Hillsdale, New Jersey, 1989

[11] Cognition and Technology Group at Vanderbilt, "Anchored Instructions and its relationship to situated cognition", Educational Researcher, 19 (1990), 2-10

[12] Cognition and Technology Group at Vanderbilt, "Technology and the design of generative learning environments", Educational Technology, 31 (1991), $34-40$

[13] J.R. Anderson, R. Farrell, R. Sauers, Learning to program in LISP, Cognitive Science, 8 (1984), 87-129

[14] A. Renkl, R. Stark, H. Gruber, H. Mandl, Learning from worked-out examples: The effects of example variability and elicited self-explanations, Contemporary Educational Psychology, 23 (1998), 90-108

[15] S. Ohlsson, Some Principles of Intelligent Tutoring. In Lawler \& Yazdani (Eds.), Artificial Intelligence and Education, 1, Ablex: Norwood, NJ, 1987, 203-238

[16] Wenger E., Artificial Intelligence and tutoring systems. Los Altos, CA. Morgan-Kaufmann, 1987 